\title{
Mapping the Distortion Function via Multivariate Analysis of Atomically Resolved Images
}

Kevin Roccapriore ${ }^{1}$, Matthew Chisholm ${ }^{1}$, Gerd Duscher ${ }^{2}$, Sergei Kalinin ${ }^{1}$ and Maxim Ziatdinov ${ }^{1}$

${ }^{1}$ Oak Ridge National Laboratory, Oak Ridge, Tennessee, United States, ${ }^{2}$ The University of Tennessee Knoxville, Knoxville, Tennessee, United States

Scanning Transmission Electron Microscopy is the primary technique used in quantitative measurement of atomic structure of materials. With the advent of aberration correction, atomic coordinates are now able to be mapped. Displacements of atomic columns from an otherwise perfect crystal lattice can reveal information pertaining to strains, polarizations, octahedral tilts, and other physical and chemical order parameter fields. Such quantitative structural characterization, however, ordinarily requires a deep understanding of the microscope-induced distortions. In this sense, we broadly categorize the distortions that are present within a STEM image into two types: scan-related distortions and material distortions. The scan distortions consist of sample drift, fly-back delays, and other behavior that is difficult to predict resulting from the scanning systems. Decoupling these two distortions to make possible accurate mapping of atomic coordinates is the goal of this paper. It is important to consider that these distortion parameters are affected by a variety of different causes such as specific scan conditions, day of the week, electronics settings, etc. Other distortion-correction methods exist such as RevSTEM ${ }^{1}$, and acquiring a set of orthogonal scan pairs ${ }^{2}$, which attempt to correct for the scan distortions. These essentially balance the difference between the fast and slow scan axes, which is a considerable improvement in obtaining more accurate atomic coordinates. However, in these cases scan distortions are not completely avoided.

To attack this problem, we begin with a physically undistorted crystal such that any distortion we do encounter must be that induced by the scanning system and potentially specimen drift. By generating an ideal crystal lattice as a comparison tool, it becomes apparent where in space the acquired STEM image suffers from distortions. Second, we acquire image stacks in order to track the atomic column positions in time. In this way, we obtain the spatiotemporal structure of the microscopic distortions which aids in separating the spatial drift of the specimen from the scan induced distortions. Using a multivariate analytical approach of these atomic column trajectories obtained in the image stacks, we find the relevant Principal Component Analysis (PCA) component, and from this, we develop the Gaussian Process ${ }^{3}$ based regression method for the quantification of the distortion function. With the knowledge of the distortion function, one can compensate for the difficult-to-understand distortions induced by the scanning system and consequently accurately determine atomic coordinates. This translates to the ability to accurately quantify strains, polarization fields, and other displaced-related phenomena. This approach is not only limited to STEM, but also is valid for any scanning-based system, as these will all also inherently suffer from a variety of scan-related distortion. The limitations of such an approach and possible further strategies are discussed. 

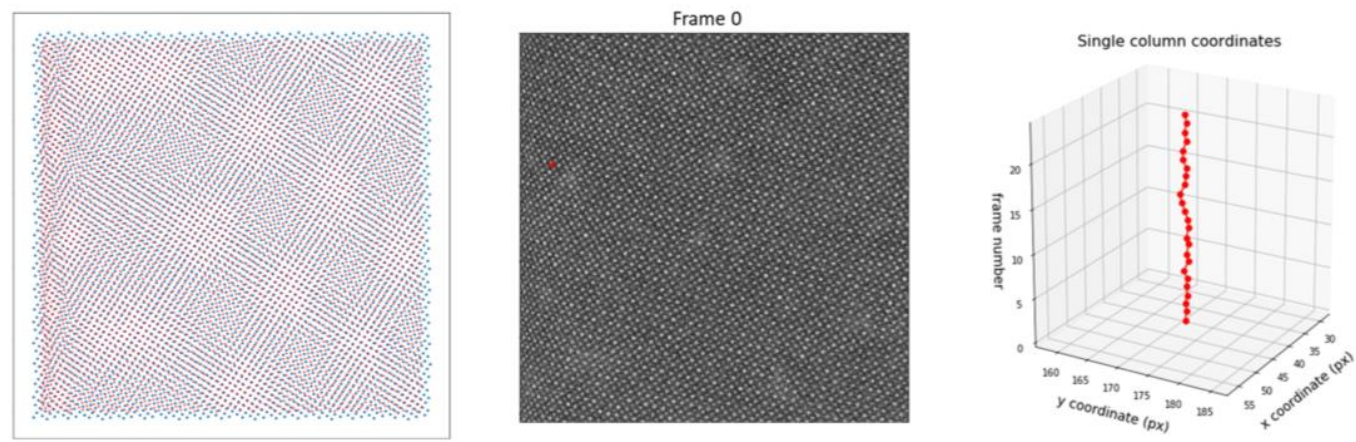

Figure 1. Experimentally found atom positions in red overlaid with ideal, non-distorted lattice positions in blue (left)); initial frame in image stack of experimental STEM image highlighting one atom (center), atomic column position indicated in center figure as a function of stack number (right).

\section{References}

1. Sang, X. \& LeBeau, J. M. Revolving scanning transmission electron microscopy: Correcting sample drift distortion without prior knowledge. Ultramicroscopy 138, 28-35 (2014).

2. Ophus, C., Ciston, J. \& Nelson, C. T. Correcting nonlinear drift distortion of scanning probe and scanning transmission electron microscopies from image pairs with orthogonal scan directions. Ultramicroscopy 162, 1-9 (2016).

3. Rasmussen, C. E. Gaussian Processes in Machine Learning. in Advanced Lectures on Machine Learning: ML Summer Schools 2003, Canberra, Australia, February 2 - 14, 2003, Tübingen, Germany, August 4 - 16, 2003, Revised Lectures (eds. Bousquet, O., von Luxburg, U. \& Rätsch, G.) 63-71 (Springer, 2004). doi:10.1007/978-3-540-28650-9_4.

\section{Acknowledgments}

This effort is based upon work supported by the U.S. Department of Energy (DOE), Office of Science, Basic Energy Sciences (BES), Materials Sciences and Engineering Division and was performed at the Oak Ridge National Laboratory's Center for Nanophase Materials Sciences (CNMS), a U.S. Department of Energy, Office of Science User Facility. 